\title{
Características de los elementos constitutivos en las guías y protocolos de algunas unidades didácticas de la práctica intermedia I, con énfasis en planeación y diseño, elaboradas por los estudiantes para profesor, en el periodo 2008(1)-2012(1) de LEBEM ${ }^{1}$
}

\author{
Features of the constituent elements in the guidelines and \\ protocols of some practice teaching units of intermediate $I$, \\ with emphasis on planning and design, developed by student \\ teachers in the period 2008 (1) -2012 (1) of LEBEM \\ Características dos elementos constitutivos das diretrizes e protocolos \\ de algumas unidades de ensino da prática de intermediário \\ I, com ênfase em planejamento e projeto, desenvolvido por \\ professores-alunos no período de 2008 (1) -2012 (1) de LEBEM
}

Recibido: mayo de 2013

Aceptado: agosto de 2013
Andrea Paola Morales Vargas ${ }^{2}$

Vivian Lucila Castillo Hernández ${ }^{3}$

Neila Sánchez Heredia ${ }^{4}$

\begin{abstract}
Resumen
En este articulo se presenta el trabajo realizado en el marco, de la Pasantía ${ }^{5}$ de Investigación "sistematizar y analizar la información dispuesta en las guías de profesor y protocolos de actividades de algunas unidades didácticas elaboradas por los estudiantes para profesores de las prácticas intermedias, de los periodos comprendidos entre el 2005 (I) y 2012 (I), para caracterizar el desarrollo del Conocimiento didáctico de contenido (CDC) de la Práctica Intermedia I con énfasis en la Planeación y Diseño, desarrollada por los estudiantes para profesor de matemáticas (EPM), de IV semestre durante los periodos 2008-I al 2012-I de la Licenciatura de Educación Básica con énfasis en Matemáticas (LEBEM), de la Universidad Distrital Francisco José de Caldas.
\end{abstract}

Palabras clave: Profesor; formación inicial de profesores; enseñanza; planificación del profesor; análisis didáctico; unidad didáctica; elementos constitutivos; diseño; EPM.

\footnotetext{
1 Artículo de Investigación

2 Universidad Distrital Francisco José de Caldas. Bogotá, Colombia. Contacto: Andre.itala.l@hotmail.com

3 Universidad Distrital Francisco José de Caldas. Bogotá, Colombia. Contacto: Vivianlu-5@hotmail.com

4 Universidad Distrital Francisco José de Caldas. Bogotá, Colombia. Contacto:nsanchez@udistrital.edu.co

5 Somos pasantes del grupo de investigación Crisálida, para optar por el título de licenciadas en educación básica con énfasis en matemáticas del proyecto curricular LEBEM.
} 


\begin{abstract}
This paper presents the work done in the framework of the Research Pasantía1 "systematize and analyze the information provided in the teacher guides and activities of some protocols of teaching units for students for teachers intermediate practices of the periods from 2005 (I) and 2012 (I), to characterize the development of pedagogical knowledge of content (CDC) Intermediate Practice I with emphasis on Planning and Design, developed by student teachers of mathematics (EPM) of IV semester during the periods 2008-I to 2012-I of the Basic Education Degree with an emphasis in Mathematics (LEBEM), University Francisco José de Caldas.
\end{abstract}

Keywords: Professor, initial teacher training, teaching, teacher planning, analysis didactic teaching unit; components; design EPM.

\title{
Resumo
}

Este artigo apresenta o trabalho desenvolvido no âmbito da Pesquisa Pasantía1 "sistematizar e analisar as informações fornecidas nos guias de professores e atividades de alguns protocolos de unidades de ensino para os alunos de professores práticas intermediários do períodos de 2005 (I) e 2012 (I), para caracterizar o desenvolvimento do conhecimento pedagógico do conteúdo (CDC) Prática I Intermediário com ênfase em planejamento e projeto, desenvolvido pelos professores dos alunos de matemática (EPM) do IV semestre durante os períodos de 2008-I e 2012-I do Curso de Educação Básica, com ênfase em Matemática (LEBEM), Universidade Francisco José de Caldas.

Palavras-chave: Professor, formação, ensino, professor de planejamento, análise de unidade de ensino didático inicial de professores; componentes; projeto EPM.

\section{Planteamiento del problema}

El plan de estudios del proyecto curricular LEBEM. (Licenciatura en educación básica con énfasis en matemáticas) se encuentra estructurado a partir de 4 ejes de formación, los cuales son: eje de pensamiento de Problemas de la Matemática escolar, eje de didáctica, eje de práctica y eje de contextos profesionales.

Para llevar a cabo esta investigación nos centramos en práctica intermedia I con énfasis en planeaciòn y diseño, del eje de práctica docente, se realiza en educación básica primaria en colegios oficiales, por EPM de cuarto semestre; esta debe tener en cuenta todos los factores que influyen en el quehacer docente, tanto el contexto y la población en la cual se va a desarrollar, el tipo de recursos que tiene a su disposición, el nivel de conocimiento y comprensión de las diferentes temáticas de los estudiantes evidenciada en la prueba diagnóstico, la forma en la cual va a trabajar el objeto matemático para que se dé un aprendizaje significativo y con sentido, para esto se tiene en cuenta la planeación y diseño de las actividades, en la que se expone: la situación fundamental, los objetivos generales y específicos, el referente teórico y metodológico, la metodología a aplicar, la guía del estudiante y los criterios de evaluación en los diferentes aspectos.

El problema está enfocado en la recolección y organización de las unidades didácticas (UD) de esta práctica, aquí se observa y/o analiza como es el diseño y la planeación, en que autores se basan. Además se quiere evidenciar como planean, como 
gestionan, cuales son los principales problemas que se le presentan a los EPM y que lo inducen a reflexionar sobre su accionar didáctico antes, durante y después de su intervención en el aula.

De acuerdo a esto surge la siguiente pregunta: ¿Cuáles son las características de los elementos constitutivos en las guías y protocolos de algunas unidades didácticas de la práctica intermedia I, con énfasis en planeación y diseño, elaboradas por los EPM, en el periodo 2008(1)-2012(1) de LEBEM? investigación teniendo en cuanta el análisis. La síntesis, la deducción etc.

Las fuentes impresas (documentos escritos) nos sirven para la investigación documental y bibliográfica. Estas dos investigaciones se deducen como iguales, en donde la investigación documental revisa y analiza libros y la bibliográfica nos sirve para una investigación documental.

\section{Metodología}

\section{Marco referencial conceptual}

El siguiente referente da cuenta de una de las lecturas realizadas sobre investigación y sistematización de información, en donde se encuentran diferentes aspectos que nos ayudan en el análisis y sistematización de las unidades didácticas

La investigación documental ${ }^{3}$ como una variante de la investigación científica el objetivo de esta es analizar diferentes fenómenos como (psicológicos, históricos etc.) utiliza técnicas precisas que aportan a la información.

Es algo que se basa en la realidad donde se observa, se reflexiona se indaga, se interpreta y se presentan datos los cuales pueden ser tabulados para obtener resultados para el desarrollo de la creación científica.

Visto en términos esta investigación la podemos caracterizar así:

Se caracteriza por la utilización de documentos, ya que se recolectan, se seleccionan, se analizan y se presentan resultados coherentes para utilizar los procedimientos lógicos y mentales de toda la
Para realizar la primera parte de esta investigación se recolectaron 72 unidades, las cuales fueron solicitadas a los profesores que las habían asesorado, a los estudiantes de LEBEM. Y en algunos casos se visitaron las instituciones en las que se había llevado a cabo la práctica; inicialmente la meta era recolectar como mínimo 10 UD por periodo, pero en este caso se recolectaron más.

El segundo paso consistió en organizar la información espacio-temporal de estas unidades en una matriz, la cual contiene los siguientes datos: periodo, profesor asesor, autores, institución, curso, pensamiento, tema, breve descripción de la UD. En la ilustración 1 se presenta una imagen que muestra la estructura de la matriz.

Como tercer paso, se realizaron fichas de identificación de cada UD (como se puede ver en la ilustración 2) en donde se identificaron los principales aspectos de cada una de estas. Y finalmente se descartaron las UD que no tenían la reflexión correspondiente a planeación y diseño dentro de sus protocolos o que la tenían incompleta, para finalmente dejar solo 15, con las cuales se va a seguir trabajando y se les va a realizar un análisis más profundo. 
Ilustración 1

\begin{tabular}{|c|c|c|c|c|c|}
\hline Periodo & Curso & Pensamiento & Tema & Modelo & Descripoión general unidad didactioa \\
\hline $2008-1$ & 5 & Metrico & $\begin{array}{l}\text { perimetro, área, conversion de } \\
\text { medidas y volumen }\end{array}$ & DECA & $\begin{array}{l}\text { Planeación: propositos generales y especificos para } \\
\text { estudiantes y profesores, descripción por momentos, criterios } \\
\text { de evaluación para estudiantes y profesores. Protocolo: } \\
\text { descripción detallada, gestión del profesor, evaluación según } \\
\text { criterios planteados, reflexión de planeación }\end{array}$ \\
\hline $2008-3$ & 0 & Númetico & $\begin{array}{l}\text { DISFRUTEMOSCON WINNE THE } \\
\text { POOHCONTANDO, ORDENANDO } \\
\text { Y AGRUPANDO } \\
\end{array}$ & DECA & $\begin{array}{c}\text { Planeacion: cada actividad incluye plan b } \\
\text { Procotoco: describe detalladamente la clase, no presenta } \\
\text { evidencias }\end{array}$ \\
\hline $2008-3$ & 3 & Metrico & La magnitud, longitud & DECA & $\begin{array}{c}\text { planeacion: muy general } \\
\text { protocolos: analiza cada aspecto de evaluacion, no presenta } \\
\text { evidencias. }\end{array}$ \\
\hline $2008-3$ & 4 & Metrico & $\begin{array}{l}\text { Magnitud amplitud a partir de los } \\
\text { giros }\end{array}$ & DECA & $\begin{array}{c}\text { Planeacion: evaluacion por momentos, muestra las actividades } \\
\text { que tendran que desarrollar los estudiantes protocolos: } \\
\text { reflexion planeacion, presenta evidencias. }\end{array}$ \\
\hline $2009-1$ & 1 & Metrico & Midiendo,miediendo & DECA & $\begin{array}{l}\text { Planeación: descripción por momentos, relación con clase } \\
\text { anterior Protocolo: enfasis en gestión del profesor, analisis de } \\
\text { planeaciony diseño, describe detalladamente la clase. }\end{array}$ \\
\hline $2009-1$ & 2 & Geométrico & $\begin{array}{l}\text { Reconociendo la geometria con mi } \\
\text { mundo }\end{array}$ & DECA & $\begin{array}{l}\text { Planeación justificación, descripción por momentos y tareas, } \\
\text { de acuerdo a cada momento. Protocolo: enfasís en gestión del } \\
\text { profesor, analisis de planeación y diseño. }\end{array}$ \\
\hline $2009-1$ & 3 & Metrico & Las magnitudes & DECA & $\begin{array}{c}\text { Planeación justificación, descripción general de la actividad, } \\
\text { indicadores paqra docentes y estudiantes. Protocolo: analisis } \\
\text { de la clase, evaluación de acuerdo a indicadores, no presenta } \\
\text { analisis de planeación y diseño }\end{array}$ \\
\hline $2009-1$ & 0 & Geométrico & Nociones de situación & DECA & $\begin{array}{c}\text { Planeación descripción por momentos y criterios de evaluación } \\
\text { para docentes y estudiantes. Protocolo: evaluación de cada } \\
\text { punto, con graficas de barras, no presenta evaluacion en } \\
\text { cuanto a planeación y diseño. }\end{array}$ \\
\hline $2009-1$ & 5 & Metrico & la medida & DECA & $\begin{array}{l}\text { Planeación: descripción por momentos, indicadores de } \\
\text { evaluación, Protocolo: descripción, evaluación según } \\
\text { indicadores, no presenta analisís de planeación y diseño. }\end{array}$ \\
\hline
\end{tabular}

Fuente: Elaboración propia

Ilustración 2

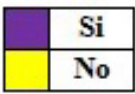

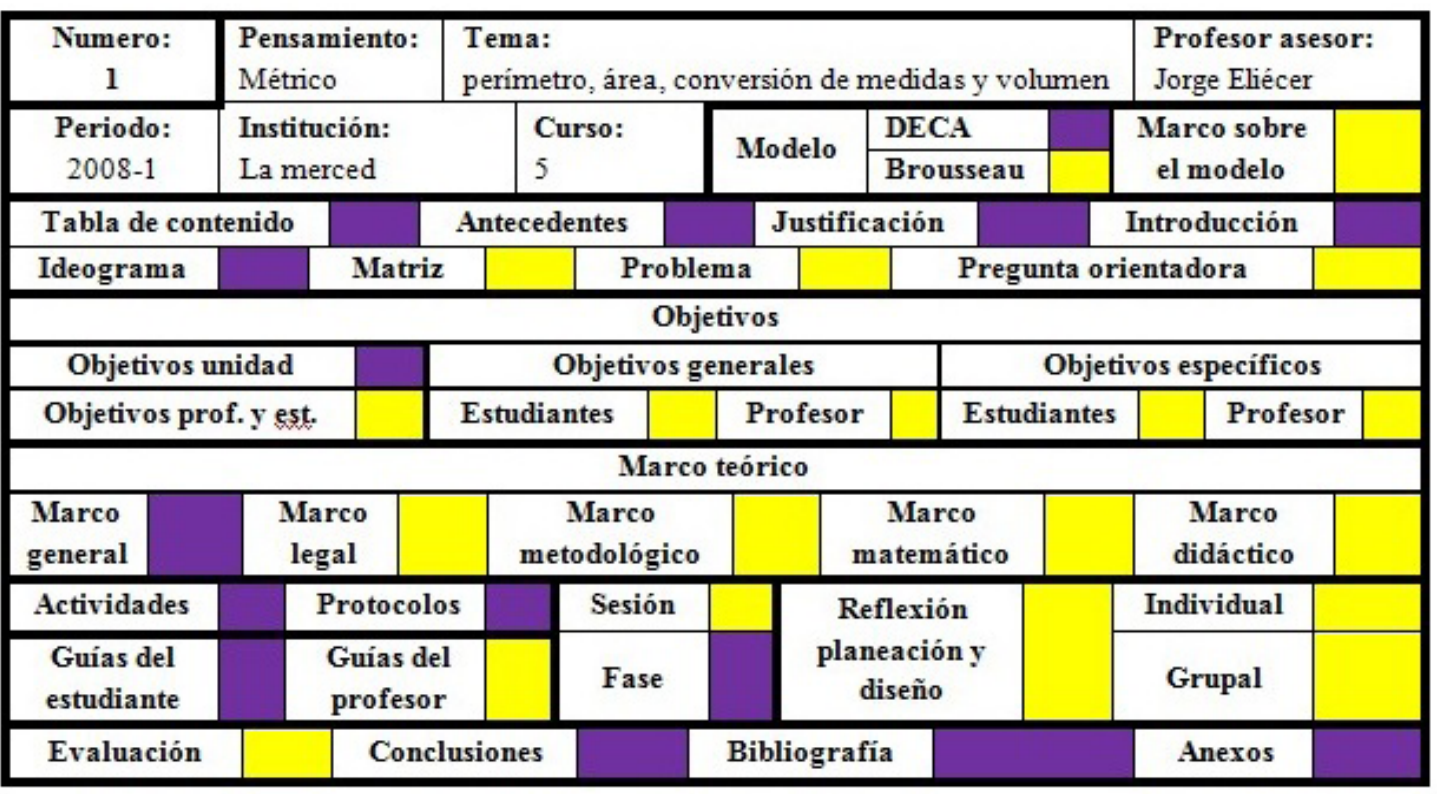

Fuente: Elaboración propia 


\section{Resultados}

Las UD recolectadas corresponden a los siguientes periodos:

Tabla 1

\begin{tabular}{|c|c|}
\hline Periodo & Cantidad \\
\hline $2008-1$ & 1 \\
\hline $2008-3$ & 3 \\
\hline $2009-1$ & 5 \\
\hline $2009-3$ & 7 \\
\hline $2010-1$ & 10 \\
\hline $2010-3$ & 3 \\
\hline $2011-1$ & 15 \\
\hline $2011-3$ & 20 \\
\hline $2012-1$ & 8 \\
\hline
\end{tabular}

Fuente: Elaboración propia

Dentro de esta investigación se encontró que a pesar de que se maneja la misma metodología, hay grandes diferencias entre las estructuras de las unidades didácticas, pues algunas contienen apartes que otras no, como la reflexión correspondiente a los protocolos sobre planeación y diseño, que es nuestro principal objetivo, pues muy pocas UD la contienen y algunas que la contienen son muy superficiales.

Después de dejar 15 UD en el primer filtro, lo cual se realizo bajo dos criterios: que tuvieran reflexión sobre planeación y diseño en los protocolos y según la cantidad de UD del periodo se dejaba la muestra correspondiente (por ejemplo en el caso de tener 15 UD de un periodo, se seleccionaron $3)$. En el siguiente filtro solo se dejaran 5 UD las cuales serán analizadas con un examen más complejo y profundo, pues cada uno de sus apartes serán detallados con una mirada de evaluadores, realizando comentarios y dando una nota final de la UD.

\section{Conclusiones}

En la recolección de la información es necesario tener en cuenta el periodo en orden cronológico de forma ascendente, ubicando el lugar donde fueron desarrolladas las practicas.

Se evidencio que todas las UD recolectadas fueron desarrolladas en grados de educación básica primaria en colegios oficiales del Distrito Capital (Bogotá).

Los temas que se desarrollaron en las UD se centran en el pensamiento numérico y desarrollo del pensamiento espacial.

\section{Referencia}

Abela, J. Técnicas de análisis de contenido. Universidad de granada. p. 34

Grupo en Ingeneria TEelematica. Modelo para la investigación documental. Título de la obra completa. Universidad del cauca. 2009. p.p.12-20.

Recopilación Lurduy. Investigación documental. p.p. 1-10.

Recopilación Lurduy. Técnicas de investigación. p.p. 1-6.

Recopilación Lurduy, Tipos de investigaciones.

Hyos, C. Un modelo para la investigación documental. p.p. 1-64. 